\title{
Development and Verification of a Precolumn Derivatization LC-MS/MS Method for the Pharmacokinetic Study of Houttuynine of Houttuynia Essential Oil
}

\author{
Yuanyuan Liu ${ }^{1,2}$, Yanfang Yang 1,2, Bangyuan Wang ${ }^{1,2}$, Renyun Wang 1,2, Jianmei Pang 1,2, Yu Jiang 1,2 \\ and Yuling Liu 1,2,*
}

check for updates

Citation: Liu, Y.; Yang, Y.; Wang, B.; Wang, R.; Pang, J.; Jiang, Y.; Liu, Y. Development and Verification of a Precolumn Derivatization LC-MS/MS Method for the Pharmacokinetic Study of Houttuynine of Houttuynia Essential Oil. Molecules 2021, 26, 2327. https:// doi.org/10.3390/molecules26082327

Academic Editor:

Domenico Trombetta

Received: 18 March 2021

Accepted: 11 April 2021

Published: 16 April 2021

Publisher's Note: MDPI stays neutral with regard to jurisdictional claims in published maps and institutional affiliations.

Copyright: (c) 2021 by the authors. Licensee MDPI, Basel, Switzerland. This article is an open access article distributed under the terms and conditions of the Creative Commons Attribution (CC BY) license (https:/ / creativecommons.org/licenses/by/ $4.0 /)$.
1 State Key Laboratory of Bioactive Substance and Function of Natural Medicines, Institute of Materia Medica, Chinese Academy of Medical Sciences \& Peking Union Medical College, Beijing 100050, China; liu20220883@163.com (Y.L.); yangyf@imm.ac.cn (Y.Y.); azqok2@163.com (B.W.); wry@imm.ac.cn (R.W.); pjmkerry@sina.com (J.P.); 17865562120@163.com (Y.J.)

2 Beijing Key laboratory of Drug Delivery Technology and Novel Formulation, Institute of Materia Medica, Chinese Academy of Medical Sciences \& Peking Union Medical College, 1 Xiannongtan Street, Beijing 100050, China

* Correspondence: ylliu@imm.ac.cn; Tel.: +86-10-6315-9373; Fax: +86-10-8928-5090

\begin{abstract}
Houttuynia essential oil (HEO) has excellent antiviral, anti-inflammatory, and other pharmacological effects, but the lack of effective analytical methods to quantify HEO in plasma has hindered its better clinical monitoring. Houttuynine (Hou) is one of the main active ingredients and quality control substances of HEO, so the pharmacokinetic study of HEO could be conducted by determining Hou blood concentration. Hou is active and not stable in plasma, which makes its blood concentration difficult to measure. In this work, a novel liquid chromatography tandem mass spectrometry (LC-MS/MS) method for Hou determination in rat blood was established that involves Hou being derivatized with 2, 4-dinitrophenylhydrazine to form a stable compound to prevent degradation. Herein, $p$-Tolualdehyde-2,4-dinitrophenylphenylhydrazone was selected as an internal standard substance and the LC-MS/MS method was evaluated for selectivity, precision, accuracy, calibration limit, matrix effect, recovery, and stability. Good linearity $\left(r^{2}=0.998\right)$ was reached in the range of 2-2000 $\mathrm{ng} / \mathrm{mL}$, and the lower limit of quantification of Hou was determined to be $2 \mathrm{ng} / \mathrm{mL}$. The mean intra-assay accuracy ranged from $77.7 \%$ to $115.6 \%$, whereas the intra-assay precision (relative standard deviation, RSD) was below $11.42 \%$. The matrix effect value for Hou in rat plasma was greater than $75 \%$, and for the internal standard (IS) it was $104.56 \% \pm 3.62 \%$. The extraction recovery of Hou were no less than $90 \%$, and for the IS it was $96.50 \% \pm 4.68 \%$. Our method is sensitive and reliable and has been successfully applied to the pharmacokinetic analysis of Hou in rats given $\mathrm{HEO}$ via gavage and injection.
\end{abstract}

Keywords: Houttuynia cordata Thunb.; houttuynine; houttuynia essential oil; LC-MS/MS; pharmacokinetic study

\section{Introduction}

Houttuynia cordata Thunb. belongs to the Saururaceae family and is a perennial herbaceous plant with a fishy smell. It is well known as both medicine and food in China, Korea, Japan, and Southeast Asia [1,2]. Its growing conditions tend to be shady and moist, and it is mainly distributed in southern areas such as in Guizhou and Wuhan in China [3]. Houttuynia cordata Thunb. has many pharmacological activities: anti-microbial, anti-inflammatory, anti-tumor, and so on [4-7]. It contains various compounds, including flavonoids, polysaccharides, alkaloids, volatiles, and fatty acids [8-10]. Among these, Houttuynia essential oil (HEO) is known to be the main active substance, which includes houttuynine (Hou), 2-undecanone, camphene, pinene, limonene, myrcene, and so on [11,12]. HEO has been widely used to treat tumors, osteoporosis, asthma, pneumonia, keratitis, and respiratory 
infections [13-15]. However, the lack of effective analytical methods to quantify HEO in plasma has hindered its better clinical monitoring. Since Hou is one of the main active ingredients [16,17] and quality control materials in HEO, the pharmacokinetic study of HEO could be conducted by determining the concentration of Hou in blood.

Gas chromatography-mass spectrometry (GC-MS), solid-phase microextraction (SPME)GC-MS, high-performance liquid chromatography (HPLC), and liquid chromatography tandem mass spectrometry (LC-MS/MS) are widely used for the detection of volatile oil content [2,3,18-22]. At present, gas chromatography (GC) is the most widely used for Hou determination [2,3], but it cannot detect Hou in blood. Moreover, Hou is a $\beta$ dicarbonyl compound with chemical activity, which is easily degraded, polymerized, oxidized [23], and not stable in plasma, making its blood concentration difficult to measure. Therefore, establishing an efficient and accurate method to quantify Hou in plasma is key to conducting pharmacokinetic studies of HEO.

Based on the determination method of sodium houttuynia ( $\mathrm{SH}$, an adducted compound of sodium bisulfite and Hou) in human plasma reported by Duan [18], Hou was derived with 2,4-dinitrophenyl hydrazine (DNPH) to form a stable compound to prevent its degradation, and the content of Hou was determined by LC-MS/MS using the internal standard (IS) method. However, we found that the method was not suitable for monitoring the changes of Hou in vivo. First, the derivatization efficiencies of the IS (benzophenone, $\mathrm{BP}$ ) and Hou are very different. As can be seen from Figure 1, Hou reacts completely within $15 \mathrm{~min}$, while the reaction of the same amount of BP is still incomplete after $8 \mathrm{~h}$. This phenomenon greatly reduced the accuracy of the Hou determination. Second, it requires the use of $20 \%$ sulfuric acid, which is corrosive and rather unsafe. Third, the pre-processing steps are complex. Therefore, it is of great importance to find an appropriate IS, optimize the plasma treatment method, and establish an efficient LC-MS/MS qualitative detection method for Hou to pave the way for the clinical application of Houttuynia cordata Thunb.

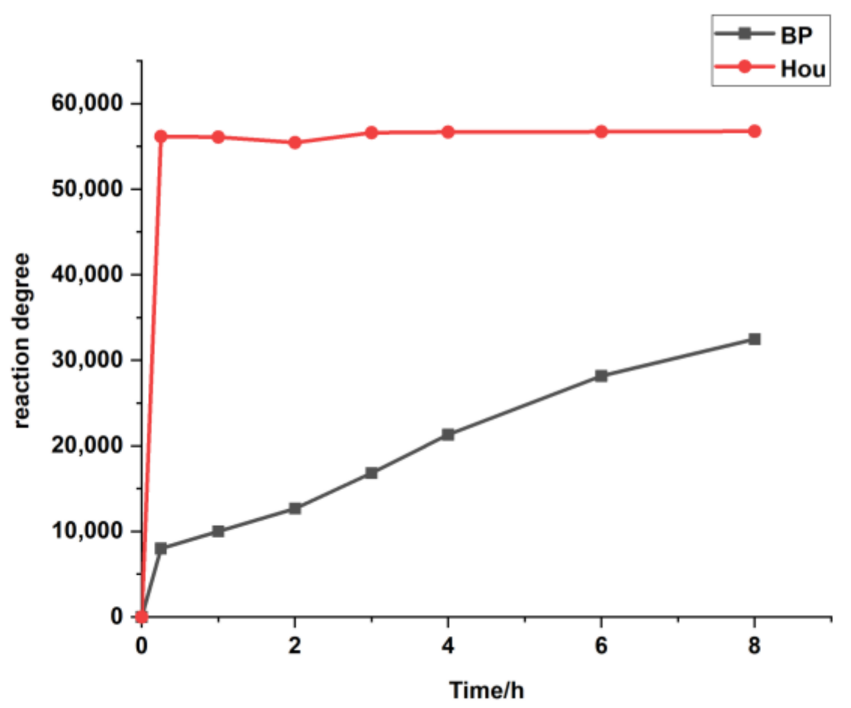

Figure 1. The response values of the derivatives in the LC-MS/MS with increasing reaction time. BP: benzophenone: Hou: houttuynine.

In this study, the hydrolyzed product of $\mathrm{SH}$, Hou, was isolated and purified. GC and nuclear magnetic resonance (NMR) were used to determine its purity and structure. $p$-Tolualdehyde-2,4-dinitrophenylhydrazone (PTD), which does not participate in the derivatization reaction, was selected as an IS to improve the accuracy of Hou detection. To improve the safety of the method, $2 \%$ hydrochloric acid $(\mathrm{HCl})$ was chosen as a catalyst. Acetonitrile was used as a solvent to remove protein in plasma samples by centrifugation. Thus, a novel LC-MS/MS method was constructed to determine Hou levels in biological samples. 
In addition, HEO has multiple components and can play a role in multiple targets; compared with a single component such as Hou or methyl nonone, it is more likely to be used as a drug. However, HEO obtained by the traditional extraction process was easily destroyed by high temperatures [24], which greatly reduces its efficacy and increases the toxic side effects such as hemolysis. Especially when given by injection, HEO can cause frequent hemolysis [25]. This research group previously used low-temperature extraction technology to prepare $\mathrm{HEO}$ with a $55 \%$ Hou content, and the safety evaluation confirmed that the safety of low-temperature extraction of HEO is much greater than that of steam distillation at the same concentration. Therefore, HEO with a 55\% Hou content was chosen as the object in the study, and the in vivo pharmacokinetics after oral administration and injection were evaluated based on the determination of Hou blood concentration using the established LC-MS/MS method.

\section{Results}

\subsection{Method Development}

Hou standard was successfully prepared by hydrolyzing $\mathrm{SH}$. Its structure and purity were determined based on GC, electron ionization mass spectrometry (ESI-MS), IR (infrared spectroscopy), NMR (nuclear magnetic resonance), and GC data. The ESI-MS showed the molecular ion peaks at $m / z 198.1[\mathrm{M}]^{+}$, this result is in accordance with previous findings [26]. The IR spectrum (Figure 2) exhibited absorption bands associated with conjugated carbonyl $\left(1633.6,1598.4 \mathrm{~cm}^{-1}\right)$, hydroxyl $\left(3418.6 \mathrm{~cm}^{-1}\right)$, methyl $\left(2954.2 \mathrm{~cm}^{-1}\right)$, and methylene $\left(2927.4 \mathrm{~cm}^{-1}\right)$, which suggested that Hou has an enol structure.

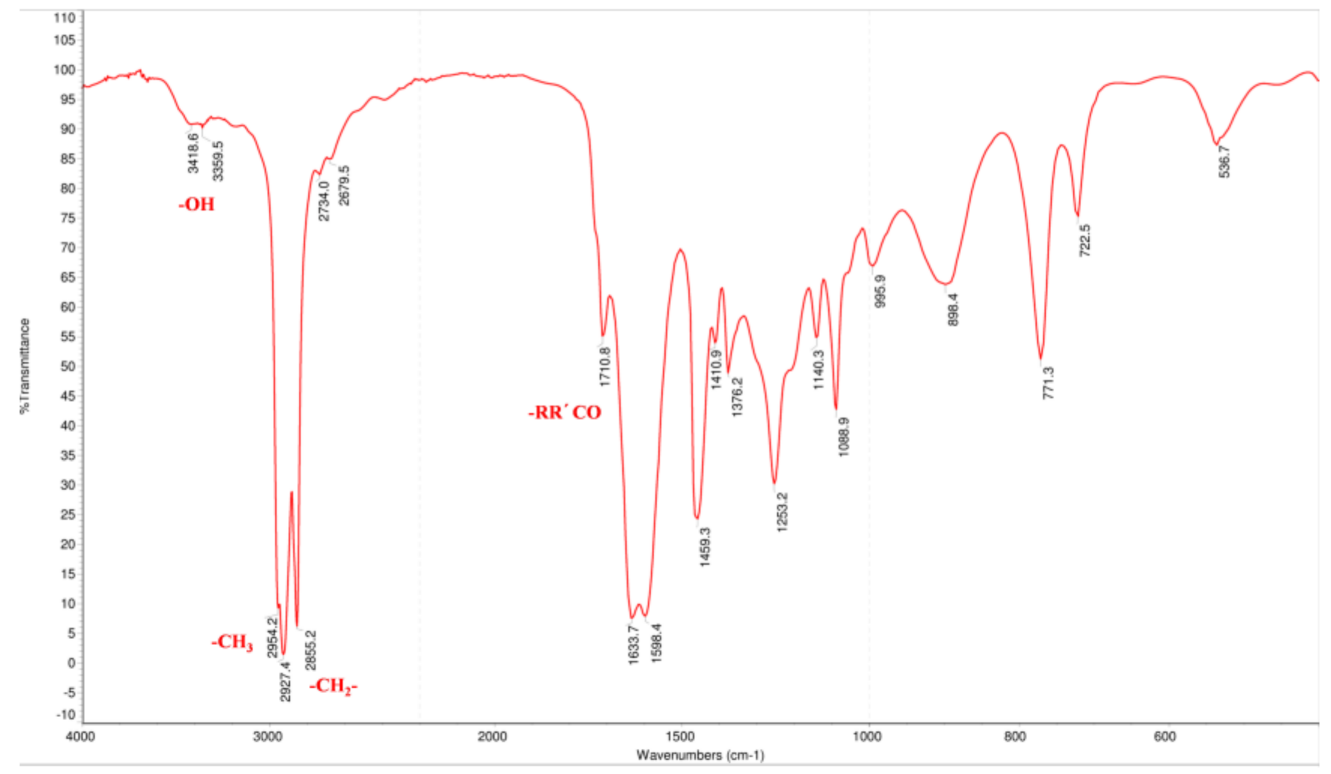

Figure 2. The IR spectrum of Hou.

The ${ }^{1} \mathrm{H}$ NMR spectrum (Figure 3) indicated an aldehyde hydrogen at $\delta_{\mathrm{H}} 7.88(1 \mathrm{H}, \mathrm{d}$, $J=4.0 \mathrm{~Hz})$, a conjugated enol hydrogen at $\delta_{\mathrm{H}} 5.49(1 \mathrm{H}, \mathrm{d}, J=4.0 \mathrm{~Hz})$, a methyl signal at $\delta_{\mathrm{H}}$ $0.83(3 \mathrm{H}, \mathrm{t}, J=7.0 \mathrm{~Hz})$, and eight methylene signals at $\delta_{\mathrm{H}} 2.31-1.21$. The ${ }^{13} \mathrm{C}$ NMR spectrum (Figure 3$)$ showed 12 carbon signals, which included one aldehyde carbon signal $\left(\delta_{C} 200.1\right)$, two double-bond carbon signals $\left(\delta_{C} 175.8,101.9\right)$, one methyl carbon signal $\left(\delta_{C} 14.3\right)$, and eight methylene carbon signals. From the above data, we inferred that the structure of the compound was 3-oxo-dodecanal, also known as Hou. 


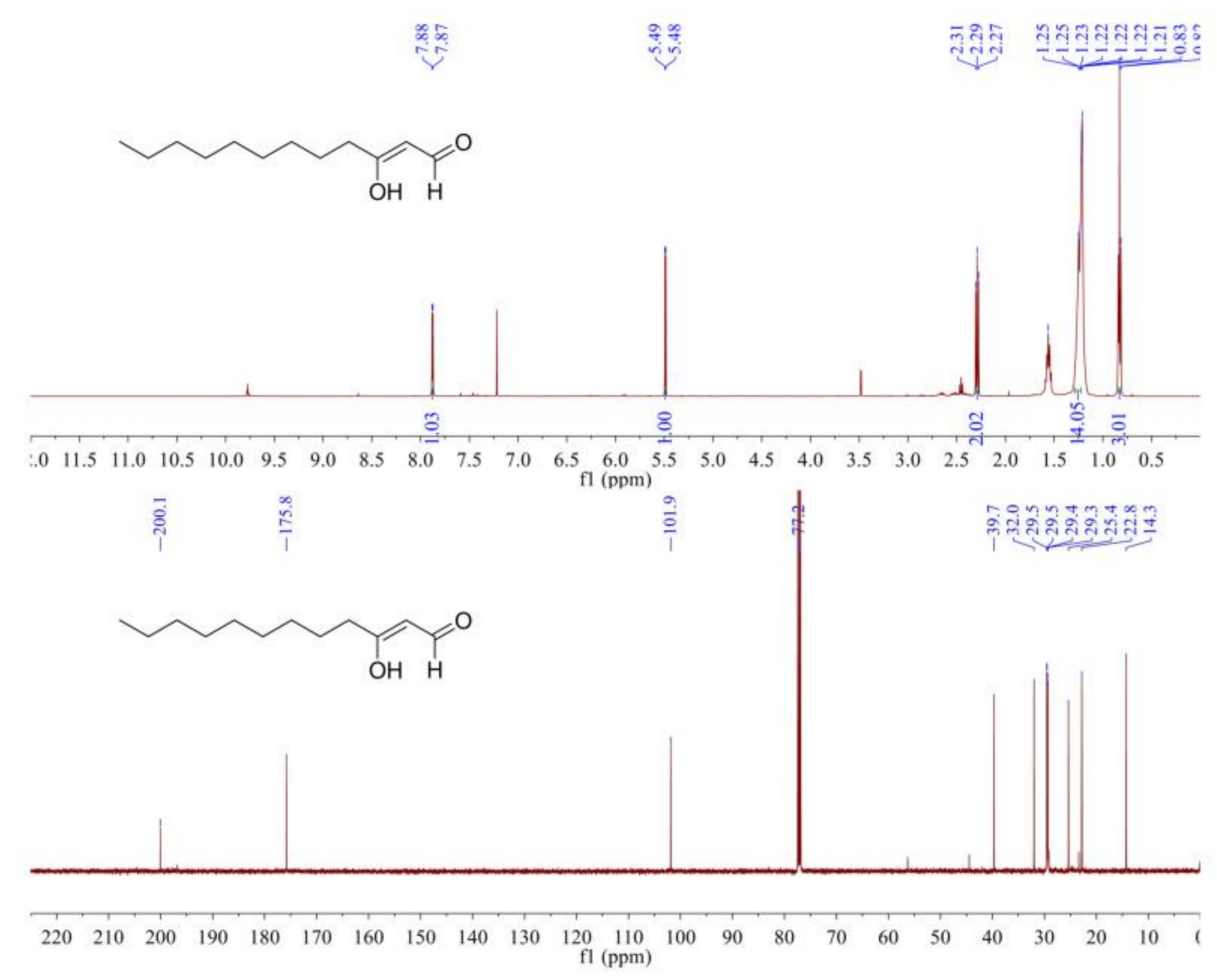

Figure 3. The ${ }^{1} \mathrm{H}$ NMR spectrum and ${ }^{13} \mathrm{C}$ NMR spectrum of $\mathrm{Hou}$ in $\mathrm{CDCl}_{3}$.

The GC spectrum in Figure 4 suggests that the prepared Hou standard was pure, in which the impurity peak was lower than the peak of Hou (retention time, $24.672 \mathrm{~min}$ ), and its relative content was $98.2 \%$ as calculated by the area normalization method.

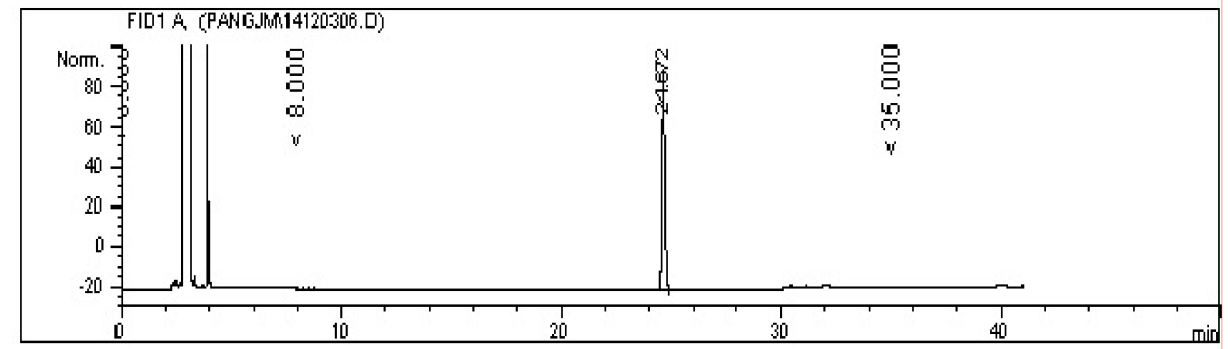

Figure 4. The GC spectrum of Hou.

We successfully obtained HEO with a 55\% Hou content according to the reported method [27]. DNPH, a reaction reagent widely used for the determination of carbonyl compounds [28-30] was selected as a derivative reagent for Hou. PTD is a stable product of formaldehyde and DNPH, and it has a molecular weight and structural characteristics similar to those of Hou-DNPH derivatives. Therefore, PTD was used as an IS to improve the detection accuracy. The equation of the Hou and DNPH reaction is shown in Figure 5.<smiles>CCCCCCCCCCCCC(=O)CCC(=O)C=CC=O</smiles>

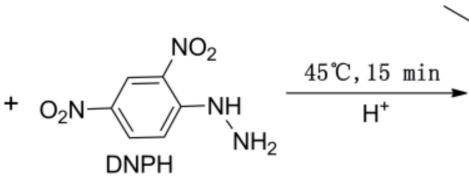<smiles>CCCCCCCCCCCCc1ccnn1-c1ccc([N+](=O)[O-])cc1[N+](=O)[O-]</smiles>
Derivative I (Hou-DNPH)

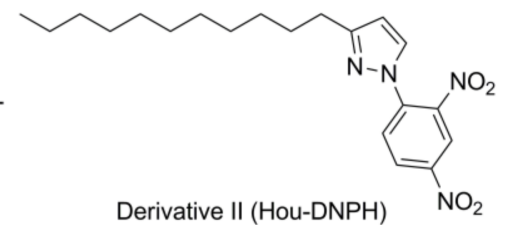

Figure 5. The equation of the Hou and 2,4-dinitrophenyl hydrazine (DNPH) reaction. 
$\mathrm{HCl}$ (at concentrations of 1-20\%) and formic acid (FA) were used in the same concentration range as the catalysts for derivatization; the results are shown in Tables 1 and 2, respectively. It can be seen from Table 1 that $\mathrm{HCl}$ significantly improved the derivatization efficiency and yield of Hou when compared to those of FA. The results for different concentrations of $\mathrm{HCl}$ shown in Table 2 suggest that $2 \% \mathrm{HCl}$ obviously improved the derivatization efficiency.

Table 1. Comparison of derivative results of $\mathrm{HCl}$ and formic acid (FA) at different concentrations. IS: internal standard.

\begin{tabular}{cccc}
\hline Catalyst & Concentration (\%) & $\begin{array}{c}\text { Response Values * of } \\
\text { the IS }\end{array}$ & $\begin{array}{c}\text { Response Values *of } \\
\text { Hou-Derivatives }\end{array}$ \\
\hline $\mathrm{HCl}$ & 20 & 9628 & 235,556 \\
$\mathrm{HCl}$ & 10 & 12,158 & 244,090 \\
$\mathrm{HCl}$ & 5 & 14,738 & 243,659 \\
$\mathrm{FA}$ & 20 & 49,170 & 52,187 \\
$\mathrm{FA}$ & 10 & 59,359 & 22,483 \\
$\mathrm{FA}$ & 5 & 61,493 & 9440
\end{tabular}

* response values mean the response strength of the compound on the mass spectrum.

Table 2. Influence of different $\mathrm{HCl}$ concentrations on the results of derivatization.

\begin{tabular}{cccc}
\hline Catalyst & Concentration (\%) & $\begin{array}{c}\text { Response Values * of } \\
\text { the IS }\end{array}$ & $\begin{array}{c}\text { Response Values * of } \\
\text { Hou-Derivatives }\end{array}$ \\
\hline $\mathrm{HCl}$ & 5 & 7867 & 379,525 \\
$\mathrm{HCl}$ & 5 & 11,956 & 348,219 \\
$\mathrm{HCl}$ & 2 & 10,764 & 423,701 \\
$\mathrm{HCl}$ & 2 & 10,146 & 419,683 \\
$\mathrm{HCl}$ & 1 & 15,631 & 23,714 \\
$\mathrm{HCl}$ & 1 & 20,048 & 52,315 \\
\hline
\end{tabular}

* response values mean the response strength of the compound on the mass spectrum.

\subsection{Method Validation}

\subsubsection{Selectivity}

The results of the selectivity evaluation indicated that there was no obvious interference signal detected at the retention times of Hou (3.288 $\mathrm{min})$ and PTD (2.308 $\mathrm{min})$ for blank plasma. Typical multiple reaction monitoring (MRM) chromatograms of blank rat plasma, plasma containing Hou and PTD, and plasma from a rat administrated with HEO with PTD are displayed in Figure 6.

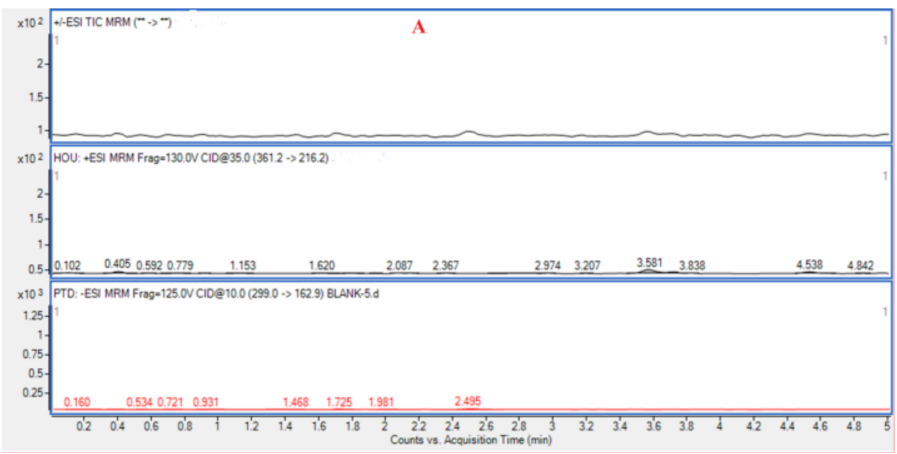

(A)

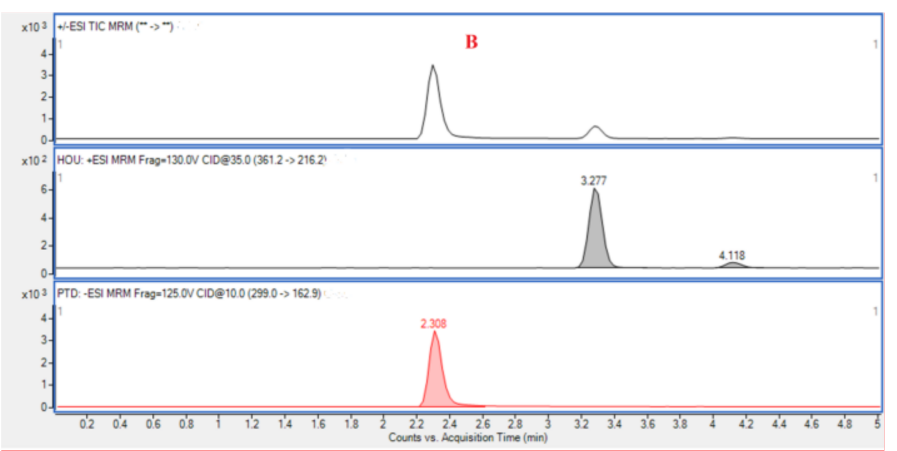

(B)

Figure 6. Cont. 


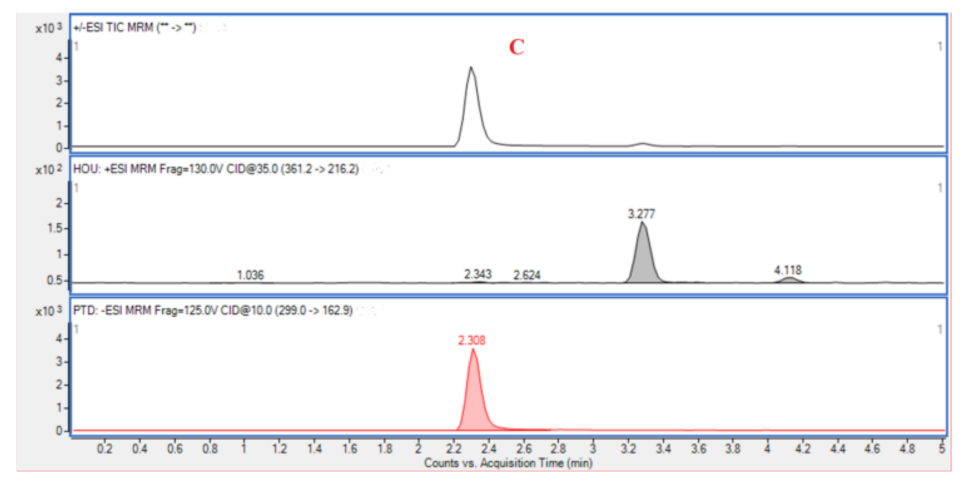

(C)

Figure 6. Typical multiple reaction monitoring (MRM) chromatograms of blank rat plasma (A), blank plasma sample spiked with analytes (B), and a plasma sample from a Houttuynia essential oil (HEO)-treated rat with $p$-Tolualdehyde-2,4dinitrophenylhydrazone (PTD) (C).

\subsubsection{Calibration Curve and Lower Limit of Quantification (LLOQ)}

The standard curve studied in the range of $2-2000 \mathrm{ng} / \mathrm{mL}$ showed good linearity, and can be specifically described as $\mathrm{Y}=0.010 \mathrm{X}-0.003472\left(n=6, \mathrm{r}^{2}=0.998\right)$, where $\mathrm{X}$ is the plasma concentration of Hou and $Y$ is the ratio of the peak area of Hou to that of the IS. The lower limit of quantification (LLOQ) of Hou was determined to be $2 \mathrm{ng} / \mathrm{mL}$ (was set at $2 \mathrm{ng} / \mathrm{mL}$ ), which is sufficient for pharmacokinetic analysis in rat plasma.

\subsubsection{Accuracy and Precision}

Intra-assay precision and accuracy results $(\mathrm{n}=6)$ for low, medium, and high concentration levels are reported in Table 3. The mean intra-assay accuracies ranged from $77.7 \%$ to $115.6 \%$, whereas the intra-assay precision, described as the relative standard deviation (RSD) of the calculated concentrations, was below $11.42 \%$. These results demonstrated the method for quantitative determination of Hou in rat plasma was reliable and repeatable.

Table 3. Accuracy and precision results of Hou derivatives $(n=6)$. RSD: relative standard deviation.

\begin{tabular}{ccccc}
\hline \multirow{2}{*}{ Analyte } & $\begin{array}{c}\text { Concentration } \\
(\mathbf{n g} / \mathbf{m L})\end{array}$ & $\begin{array}{c}\text { Measurement } \\
(\mathbf{n g} / \mathbf{m L})\end{array}$ & Accuracy (\%) & RSD (\%) \\
\cline { 3 - 5 } & 10 & $11.56 \pm 1.32$ & 115.6 & 11.42 \\
Hou- & 50 & $38.84 \pm 2.04$ & 77.7 & 5.25 \\
Derivatives & 200 & $189.33 \pm 21.02$ & 94.7 & 11.10 \\
\hline
\end{tabular}

\subsubsection{Matrix Effects and Recovery}

The matrix effect values for Hou at $10 \mathrm{ng} / \mathrm{mL}, 50 \mathrm{ng} / \mathrm{mL}, 200 \mathrm{ng} / \mathrm{mL}$, and $2000 \mathrm{ng} / \mathrm{mL}$ were $98.44 \% \pm 13.21 \%, 76.58 \% \pm 8.17 \%, 86.84 \% \pm 8.74 \%$, and $94.32 \% \pm 10.69 \%$, respectively; the matrix effect for the IS at $33.33 \mu \mathrm{g} / \mathrm{mL}$ was $104.56 \% \pm 3.62 \%$, which indicated that the co-eluting endogenous substances did not influence the ionization of the analytes. The extraction recoveries of Hou at $10 \mathrm{ng} / \mathrm{mL}, 50 \mathrm{ng} / \mathrm{mL}, 200 \mathrm{ng} / \mathrm{mL}$, and $2000 \mathrm{ng} / \mathrm{mL}$ in rat plasma were $91.32 \% \pm 20.75 \%, 94.11 \% \pm 8.70 \%, 110.70 \% \pm 13.30 \%$, and $95.38 \pm 17.61 \%$, respectively, with RSD \% values below $22.72 \%$, and the recovery of the IS was $96.50 \% \pm 4.68 \%$, with an $\mathrm{RSD} \%$ value of $4.85 \%$, which indicated that the method established to treat plasma samples has no effect on determination results and is feasible.

\subsubsection{Stability}

The stability was evaluated at low, medium, and high concentration levels $(n=6)$ and the results are presented in Table 4 . The data demonstrate that the Hou derivatives are stable for $8 \mathrm{~h}$ in plasma at room temperature $\left(25^{\circ} \mathrm{C}\right)$. 
Table 4. Stability results of Hou derivatives in plasma $(n=6)$.

\begin{tabular}{ccccc}
\hline \multirow{2}{*}{ Analyte } & $\begin{array}{c}\text { Concentration } \\
(\mathbf{n g} / \mathbf{m L})\end{array}$ & $\begin{array}{c}\text { Measurement } \\
\mathbf{( n g / m L )}\end{array}$ & Accuracy (\%) & RSD (\%) \\
\cline { 3 - 5 } & 10 & $8.98 \pm 0.71$ & 89.8 & 7.85 \\
Hou- & 50 & $45.87 \pm 1.46$ & 91.7 & 3.18 \\
Derivatives & 200 & $172.16 \pm 10.19$ & 86.1 & 5.92 \\
\hline
\end{tabular}

Taken together, the results demonstrate that the LC-MS/MS method established in this work is reliable, sensitive, and reproducible and can be used for pharmacokinetic analysis of Hou in rat plasma.

\subsection{Pharmacokinetic Study}

The pharmacokinetics of HEO administrated to rat via gavage and injection were evaluated based on the determination of Hou blood concentration by the established LCMS/MS approach. Furthermore, the parameters of the pharmacokinetics were calculated by a non-compartmental model using the Drug and Statistics Software (DAS), version 3.2.8. Mean plasma concentration-time profiles are exhibited in Figure 7, and the key pharmacokinetic parameters are calculated and listed in Table 5 . The results showed that the maximum plasma concentration $\left(C_{\max }\right)$ of Hou was $222.48 \mathrm{ng} / \mathrm{mL}$, the AUC was $2451.837 \mu \mathrm{g} / \mathrm{L} \times \mathrm{h}$, the absolute bioavailability was $7.08 \%$ in oral administration, which also suggested that $\mathrm{HEO}$ can be absorbed orally to some extent, but its absolute bioavailability is lower than that of the injectable drug.

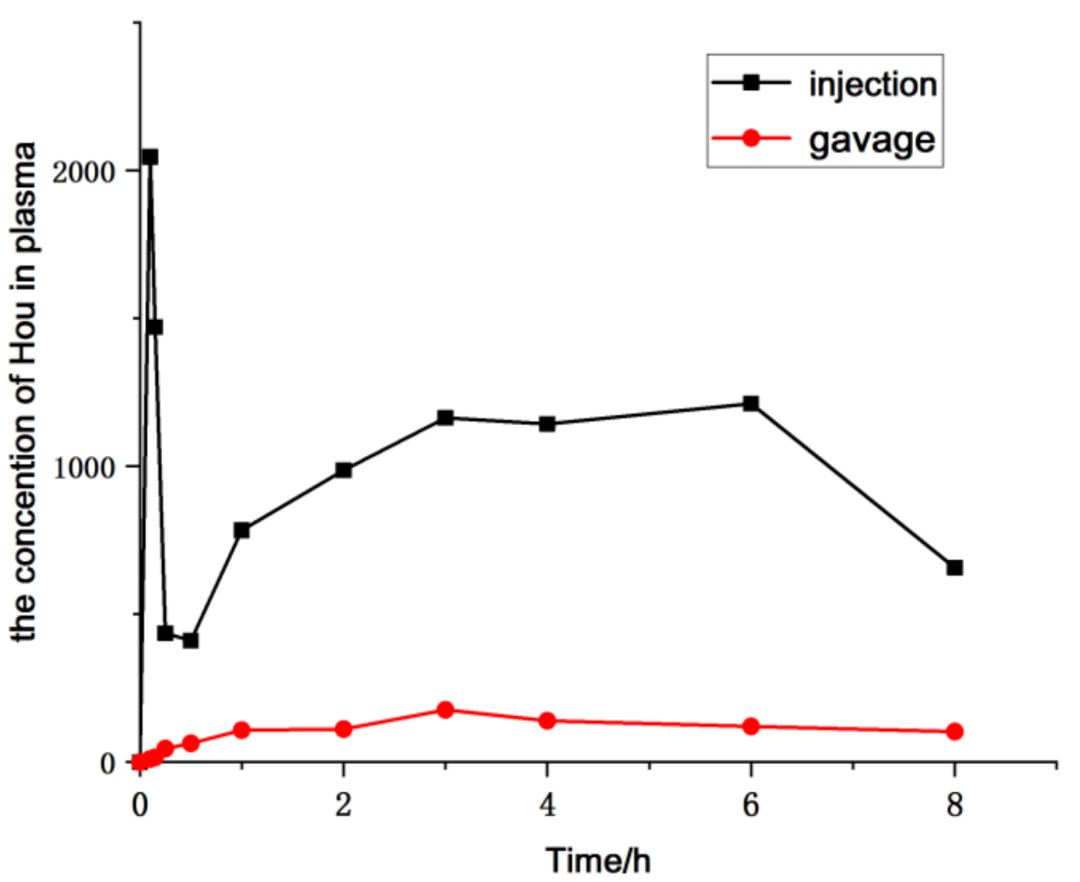

Figure 7. Mean plasma concentration-time profile of Hou in rats after a single administration of HEO by different administration forms $(n=6)$. 
Table 5. Blood concentration of Hou after a single administration of HEO by different administration forms $(n=6)$.

\begin{tabular}{cccc}
\hline Parameter & Unit & Injection & Gavage \\
\hline$T_{1 / 2}$ & $\mathrm{~h}$ & $5.987 \pm 0.52$ & $6.45 \pm 0.94$ \\
$T_{\max }$ & $\mathrm{h}$ & $0.033 \pm 0.012$ & $3.71 \pm 1.07$ \\
$C_{\max }$ & $\mu \mathrm{g} / \mathrm{L}$ & $2010 \pm 350$ & $222.48 \pm 20.38$ \\
$\mathrm{AUC}(0-\mathrm{t})$ & $\mu \mathrm{g} / \mathrm{L} \times \mathrm{h}$ & $32,693.2 \pm 1320$ & $2451.79 \pm 300.01$ \\
$\mathrm{AUC}(0-\infty)$ & $\mu \mathrm{g} / \mathrm{L} \times \mathrm{h}$ & $34,630.466 \pm 1436$ & $2451.837 \pm 432.74$ \\
$\mathrm{MRT}(0-\mathrm{t})$ & $\mathrm{h}$ & $7.575 \pm 1.21$ & $8.914 \pm 2.73$ \\
$\mathrm{MRT}(0-\mathrm{t})$ & $\mathrm{h}$ & $9.153 \pm 0.98$ & $11.594 \pm 1.85$ \\
$V_{\mathrm{z}}$ & $\mathrm{L} / \mathrm{kg}$ & $5.611 \pm 0.97$ & $85.81 \pm 5.74$ \\
$\mathrm{CLz}$ & $\mathrm{L} / \mathrm{h} \cdot \mathrm{kg}$ & $0.638 \pm 0.086$ & $8.856 \pm 3.68$ \\
$F$ & $\%$ & 100 & 7.08 \\
\hline
\end{tabular}

\subsection{Safety Evaluation}

The results showed that the animals in the intravenous administration group had reduced activity, lassitude, black tail, and hemolysis in blood samples, while there was no abnormal condition and no hemolysis in blood samples in the oral administration group. Those results indicate that the oral administration is safer and has potential as a drug delivery method. Therefore, the urgent problem to be solved in future research is to develop an appropriate new delivery system to improve the efficacy of HEO and improve the bioavailability of Hou.

\section{Materials and Methods}

\subsection{Materials}

H. cordata Thunb was provided by Yichang Jiahao Ecological Agriculture Development Co., Ltd. (Wuhan, China). Sodium houttuynia was obtained from Beijing Saige BioPharmaceutical Co., Ltd. (Beijing, China). DNPH and PTD were obtained from Aladdin Reagent (Shanghai) Co., Ltd. (Shanghai, China). Heparin sodium was provided from Wuhan Yuancheng Co-created Technology Co., Ltd. (Wuhan, China). Acetonitrile and MS-grade FA were purchased from Thermo Fisher Scientific (Fair Lawn, NJ, USA).

Sprague-Dawley (SD) male rats (200 $\pm 30 \mathrm{~g})$ were supplied by Beijing HFK Biotechnology Co. Ltd. (Beijing, China). The animal experiments met the rules set by the Ethics Committee of the Experimental Animal Center of Beijing E-Zhuang Biomedical Park, and the approval number is 2019 S019.

\subsection{Preparation of the Hou Standard}

Hou was prepared by hydrolyzing $\mathrm{SH}$ in a $0.01 \mathrm{M} \mathrm{Na}_{2} \mathrm{CO}_{3}$ solution. In short, $\mathrm{SH}(2.0 \mathrm{~g})$ was weighted precisely, transferred into a conical flask, and dissolved in a $0.01 \mathrm{M} \mathrm{Na}_{2} \mathrm{CO}_{3}$ solution. The system was stirred in an ice water bath for $15 \mathrm{~min}$ and was then extracted with dichloromethane. After vacuum drying (Gardner Denver, Karatasstr, Germany), Hou was obtained and was stored at $-80{ }^{\circ} \mathrm{C}$ for further utilization and characterization.

\subsection{Preparation of HEO}

HEO was extracted and determined according to the reported method in reference [26]. Briefly, fresh Houttuynia cordata was cut into $1 \mathrm{~cm}$ lengths, soaked with ethyl acetate (the ratio of solid to liquid was 1:1.5), extracted by ultrasonication for $30 \mathrm{~min}$, sealed, placed in a sunless place for 3 days, and concentrated to obtain crude HEO. After ethanol sinking, $n$-hexane extraction, and macroporous resin column chromatography, the HEO for pharmacokinetic and pharmacodynamic studies was obtained and analyzed by an Agilent 6890N series GC system with an Agilent DB-5 capillary column GC (30 m ×0.25 mm, $0.25 \mu \mathrm{m}$, Agilent Technologies, Santa Clara, CA, USA) for separation, and a hydrogen flame ionization detector (FID) was used for quantitative analysis [16]. 


\subsection{LC-MS/MS Instrumentation and Conditions}

We used a 6410B triple quadrupole LC-MS/MS system (Agilent, MA, USA) consisting of an Agilent 1200 RRLC system (Agilent Co.) coupled to a triple quadrupole MS analyzer with an ESI source. For chromatographic separations, we used an Eclipse XDB-C18 column $(150 \times 3.0 \mathrm{~mm}, 5 \mu \mathrm{m})$ (Agilent Technologies, Santa Clara, CA, USA). The mobile phase, consisting of acetonitrile/water/FA (90:10:0.1, $v / v / v)$, was used in the gradient elution procedure at a flow rate of $0.5 \mathrm{~mL} / \mathrm{min}$. Ten-microliter aliquots of the sample solution were injected for analysis. The separation run was finished within $5 \mathrm{~min}$ of sample injection at $25{ }^{\circ} \mathrm{C}$.

Quantification was performed in an MRM mode using ESI in the negative ion mode: for PTD, IS, $m / z$ 299- $m / z 162.9$ (fragmentor, $130 \mathrm{~V}$; collision energy, $35 \mathrm{eV}$ ); the optimized ion mode for Hou-DNPH was $m / z$ 361.2- $m / z 216.2$ (fragmentor, $125 \mathrm{~V}$; collision energy, $10 \mathrm{eV}$ ). The optimum operating parameters were as follows: drying gas temperature, $300{ }^{\circ} \mathrm{C}$; drying gas flow, $8 \mathrm{~L} / \mathrm{min}$; nebulizer pressure, $45 \mathrm{psi}$; and capillary voltage, $3.55 \mathrm{kV}$. Data were acquired and processed using the Mass Hunter workstation (Agilent).

\subsection{Preparation of Stock Solutions and Calibration Standards}

PTD was used as an IS for the analyses, and BP was used as another IS for comparison. Hou and BP-Hou were dissolved in acetonitrile to form stock solutions of $1 \mathrm{mg} / \mathrm{mL}$. Then, working solutions of $12-12,000 \mathrm{ng} / \mathrm{mL}$ were prepared from the stock solution and stored at $4{ }^{\circ} \mathrm{C}$.

A certain amount of PTD was diluted in acetonitrile under ultrasonication to obtain working solutions of $200 \mu \mathrm{g} / \mathrm{mL}$ and $100 \mu \mathrm{g} / \mathrm{mL}$. The derivative reagent, DNPH, was prepared by dissolving DNPH in acetonitrile containing $2 \%$ hydrochloric acid followed by ultrasonication to obtain a working solution of $2 \mathrm{mg} / \mathrm{mL}$.

\subsection{Plasma Sample Pretreatment}

Blank rat plasma $(100 \mu \mathrm{L})$, PTD $(200 \mu \mathrm{g} / \mathrm{mL})$, Hou working solution $(50 \mu \mathrm{L})$, and DNPH solution $(100 \mu \mathrm{L})$ were added to Eppendorf $(\mathrm{EP})$ tubes $(1.5 \mathrm{~mL})$, vortexed for a few minutes, and shaken in a $45^{\circ} \mathrm{C}$ water bath oscillator for $15 \mathrm{~min}$. Then, the system was centrifuged at 14,000 rpm, and the supernatant was removed. The above centrifugation was repeated, and the plasma samples of Hou were obtained.

\subsection{Method Validation}

A full validation of the method was performed in accordance with the Guidance for Industry Bioanalytical Method Validation [31]. The validation method was conducted for Hou in plasma according to the validation rules for biological analysis methods developed by the Food and Drug Administration (FDA). This analytical method was validated based on specificity, linearity, precision, accuracy, recovery, and stability.

\subsubsection{Selectivity}

The selectivity of the method was conducted by comparing the blank plasma samples to plasma containing Hou and PTD, and plasma from HEO-treated rats with PTD. Finally, whether the endogenous substances from blank plasma interfered the analytes or not was also studied.

\subsubsection{Calibration Curve}

Calibration curves of Hou were performed from 2 to $2000 \mathrm{ng} / \mathrm{mL}$. Linearity was determined by plotting the ratio of the peak area of the analytes to that of PTD (IS) versus the analyte concentrations and applying least-squares linear regression. A correlation coefficient $\left(\mathrm{r}^{2}\right)$ higher than 0.99 was identified as linearity. 


\subsubsection{Precision and Accuracy}

The intra-assay precision and accuracy were tested. In this work, quality control (QC) samples of plasma at high, middle, and low concentrations (six parallels at each concentration) on a single day were studied. The variability of determination was expressed as the $\mathrm{RSD} \%$, and the accuracy was expressed as the relative error (RE\%). Herein, the accuracy data within $\pm 15 \%$ RE from the nominal values and a precision data within $\pm 15 \%$ RSD were acceptable. However, the precision and accuracy data of the LLOQ should be within $\pm 20 \%$.

\subsubsection{Matrix Effect and Extraction Recovery}

The matrix effect was determined at three $\mathrm{QC}$ concentrations by comparing peak responses of post-extraction blank plasma $(n=6)$ spiked with QC samples with those of neat standard solutions. The extraction recovery was measured by comparing the responses of analytes obtained from extracted samples with blank plasma extracts spiked with QC samples. Meanwhile, the matrix effect and extraction recovery of the IS with a single concentration were also investigated.

\subsubsection{Stability}

The stability of Hou-DNPH in plasma at high, middle, and low concentrations $(n=6$ for each concentration) was investigated by keeping the samples at room temperature $\left(25^{\circ} \mathrm{C}\right)$ for $8 \mathrm{~h}$.

\subsection{Pharmacokinetic Study}

\subsubsection{Animal Study}

Twelve male SD rats (200 $\pm 30 \mathrm{~g})$ were kept in an appropriate environment for three days before the start of the experiment. Before drug administration, the rats were fasted except for free water access for $12 \mathrm{~h}$. All animals were randomly divided into two groups with six animals in each group. Blood samples $(0.2 \mathrm{~mL})$ were withdrawn at specific time intervals $(0 \mathrm{~h}, 5 \mathrm{~min}, 10 \mathrm{~min}, 15 \mathrm{~min}, 30 \mathrm{~min}, 1 \mathrm{~h}, 2 \mathrm{~h}, 3 \mathrm{~h}, 4 \mathrm{~h}, 6 \mathrm{~h}, 8 \mathrm{~h}, 12 \mathrm{~h}$, and $24 \mathrm{~h}$ ) after administration in heparinized tubes. The samples were immediately centrifuged at $3000 \mathrm{rpm}$, and kept at $-20^{\circ} \mathrm{C}$ in a refrigerator.

\subsubsection{Plasma Sample Preparation}

Plasma samples $(100 \mu \mathrm{L})$ were mixed with $100 \mu \mathrm{L}$ of DNPH $(2 \mathrm{mg} / \mathrm{mL}), 50 \mu \mathrm{L}$ of IS (PTD, $200 \mu \mathrm{g} / \mathrm{mL}$ ), and $150 \mu \mathrm{L}$ acetonitrile (containing $2 \% \mathrm{HCl}$ ) with uniform mixing, followed by vigorous vortexing for $3 \mathrm{~min}$ to precipitate the protein. Then, all samples were shaken in a water bath oscillator for $15 \mathrm{~min}$ at $45^{\circ} \mathrm{C}$. The solutions were then centrifuged. The centrifugation step was repeated once, and plasma samples of Hou were obtained and subjected to LC-MS/MS analysis.

\subsubsection{Safety Evaluation}

The safety evaluation was conducted by observing the living status and hemolysis of the above pharmacokinetic experimental animals after administration.

\subsubsection{Data Analysis}

Pharmacokinetic parameters including AUC $(0-\mathrm{t})$, AUC $(0-\infty)$, MRT $(0-\mathrm{t})$, MRT $(0-\infty)$, $\mathrm{CL}_{z}$ and so on, were calculated using the DAS (version 3.2.8, Chinese Pharmacological Association, Beijing, China). The $C_{\max }$ was obtained from the concentration-time curve. The area under the plasma concentration-time curve from zero to the time of the last measurable sample AUC $(0-t))$, area under the plasma concentration-time curve from zero to infinity AUC $(0-\infty)$, mean residence time from zero to the time of the last measurable sample $\left(\mathrm{MRT}_{0-\mathrm{t}}\right)$, mean residence time from zero to the time of infinity $\left(\mathrm{MRT}_{0-\infty}\right)$, and $\mathrm{CL}_{\mathrm{z}}$ were calculated using the DAS. 


\section{Conclusions}

We developed a novel LC-MS/MS method for Hou determination in biological samples. The method involves derivatization with DNPH. PTD is used as an IS and $2 \% \mathrm{HCl}$ as a catalyst. The method was demonstrated to be selective, linear, precise, and accurate for the determination of Hou. Good linearity $\left(r^{2}=0.998\right)$ was reached in the range of 2-2000 ng/mL, and the LLOQ of Hou was determined to be $2 \mathrm{ng} / \mathrm{mL}$. The mean intra-assay accuracy ranged from $77.7 \%$ to $115.6 \%$, whereas the intra-assay precision (RSD) was below $11.42 \%$. The matrix effect value for Hou in rat plasma was greater than $75 \%$, and for the IS it was $104.56 \% \pm 3.62 \%$. The extraction recovery of Hou was no less than $90 \%$, and for the IS it was $96.50 \% \pm 4.68 \%$. Our new method was successfully applied to the pharmacokinetic study of HEO administrated to rats via gavage and injection. The results showed that the $\mathrm{HEO}$ can be absorbed orally to some extent, but the absolute bioavailability is lower than that of the injectable drug. The safety results of the two administration modes suggested that oral administration is safer and has the potential for drug development. Based on the results, an appropriate new delivery system should be developed for maximizing the efficacy of HEO and thus improving the bioavailability of Hou.

Author Contributions: Y.L. (Yuling Liu) designed the experiment projects and directed the manuscript; Y.Y. revised the manuscript; Y.L. (Yuanyuan Liu) prepared HEO and Hou, established the pharmacokinetic detection methods, and conducted HEO pharmacokinetic studies; B.W. and J.P. were involved in optimizing the test method; R.W. and Y.J. contributed to the preparation of the HEO and Hou. All authors have read and agreed to the published version of the manuscript.

Funding: National Megaproject for Innovative drugs (No. 2018ZX09711001-002-005 and 2018ZX09721003009-001) of the Chinese government and CAMS Innovation Fund for Medical Sciences (No. CIFMS2019-12M-1-005).

Institutional Review Board Statement: The animal experiments conducted met the rules set by the Ethics Committee of the Experimental Animal Center of Beijing E-Zhuang Biomedical Park (approval number: 2019S019).

Informed Consent Statement: Not applicable.

Data Availability Statement: The data presented in this study are available on requested from the authors.

Acknowledgments: We are grateful for the financial support from the National Megaproject for Innovative drugs of the Chinese government as well as the CAMS Innovation Fund for Medical Sciences.

Conflicts of Interest: The authors declare no conflict of interest.

Sample Availability: Samples in this study can be checked with the author, Yuanyuan Liu.

\section{References}

1. Kumar, M.; Prasad, S.K.; Hemalatha, S. A current update on the phytopharmacological aspects of Houttuynia cordata Thunb. Pharmacogn. Rev. 2014, 8, 22-35. [CrossRef]

2. Shingnaisui, K.; Dey, T.; Manna, P.; Kalita, J. Therapeutic potentials of Houttuynia cordata Thunb. against inflammation and oxidative stress: A review. J. Ethnopharmacol. 2018, 220, 35-43. [CrossRef] [PubMed]

3. Fu, J.G.; Dai, L.; Lin, Z.; Lu, H.M. Houttuynia cordata Thunb: A review of phytochemistry and pharmacology and quality control. Chin. Med. 2013, 4, 101-123.

4. Cheng, D.Q.; Sun, L.; Zou, S.Y.; Chen, J.; Mao, H.Y.; Zhang, Y.J.; Liao, N.B.; Zhang, R.H. Antiviral Effects of Houttuynia cordata polysaccharide extract on murine norovirus-1 (MNV-1) —A human norovirus surrogate. Molecules 2019, 24, 1835. [CrossRef] [PubMed]

5. Her, Y.; Lee, T.K.; Ahn, J.H.; Lim, S.S.; Kang, B.G.; Park, J.S.; Kim, B.; Sim, H.; Lee, J.-C.; Kim, H.S.; et al. Chemical composition of a novel distillate from fermented mixture of nine anti-inflammatory herbs and its UVB-protective efficacy in mouse dorsal skin via attenuating collagen disruption and inflammation. Molecules 2021, 26, 124. [CrossRef]

6. $\quad$ Lau, K.M.; Lee, K.M.; Koon, C.M.; Cheung, S.F.; Lau, C.P.; Ho, H.-M.; Lee, M.Y.-H.; Au, S.W.-N.; Cheng, C.-H.K.; Lau, C.B.-S.; et al Immunomodulatory and anti-SARS activities of Houttuynia cordata. J. Ethnopharmacol. 2008, 118, 79-85. [CrossRef] [PubMed]

7. Subhawa, S.; Chewonarin, T.; Banjerdpongchai, R. The Effects of Houttuynia cordata Thunb and Piper ribesioides Wall Extracts on Breast Carcinoma Cell Proliferation, Migration, Invasion and Apoptosis. Molecules 2020, 25, 1196. [CrossRef] 
8. Nuengchamnong, N.; Krittasilp, K.; Ingkaninan, K. Rapid screening and identification of antioxidants in aqueous extracts of Houttuynia cordata using LC-ESI-MS coupled with DPPH assay. Food Chem. 2009, 117, 750-756. [CrossRef]

9. Rebickova, K.; Bajer, T.; Silha, D. Chemical composition and determination of the antibacterial activity of essential oils in liquid and vapor phases extracted from two different southeast Asian herbs-Houttuynia cordata (saururaceae) and persicaria odorata (polygonaceae). Molecules 2020, 25, 2432. [CrossRef]

10. Yang, L.; Jiang, J.G. Bioactive components and functional properties of Hottuynia cordata and its applications. Pharm. Biol. 2009, 47, 1154-1161. [CrossRef]

11. Xu, Q.S. Research status of volatile oil from Houttuynia cordata Thunb. Pharm. Ind. China 2014, 4, 15-18.

12. Zeng, H.Y.; Jiang, L.J.; Zhang, Y.C. Chemical constituents of volatile oil from Houttuynia cordata Thunb. J. Plant Resour. Environ. 2003, 12, 50-52.

13. Yang, L.S.; Ji, W.W.; Zhong, H.; Wang, L.Y.; Zhu, X.L.; Zhu, J. Anti-tumor effect of volatile oil from Houttuynia cordata Thunb. on HepG2 cells and HepG2 tumor bearing mice. RSC Adv. 2019, 9, 31517-31526. [CrossRef]

14. Li, W.F.; Fan, T.; Zhang, Y.M.; Fan, T.; Zhou, P.; Niu, X.F.; He, L.C. Houttuynia cordata Thunb. volatile oil exhibited anti-inflammatory effects in vivo and inhibited nitric oxide and tumor necrosis factor- $\alpha$ production in LPS-stimulated mouse peritoneal macrophages in vitro. Phytother. Res. 2013, 27, 1629-1639. [CrossRef] [PubMed]

15. Chen, J.; Wang, W.; Shi, C.; Fang, J. A comparative study of sodium houttuyfonate and 2-undecanone for their in vitro and in vivo anti-inflammatory activities and stabilities. Int. J. Mol. Sci. 2014, 15, 22978-22994. [CrossRef]

16. Oh, S.Y. An effective quality control of pharmacologically active volatiles of Houttuynia cordata Thunb by fast gas chromatographysurface acoustic wave sensor. Molecules 2015, 20, 10298-10312. [CrossRef] [PubMed]

17. Wang, B.Y.; Yang, Y.F.; Pang, J.M.; Liu, Z.H.; Jiang, L.M.; Liu, Y.L. Simultaneous quantification of nine volatile oils in Houttuynia cordata extracts by GC. China Pharm. 2019, 22, 1261-1264.

18. Duan, X.T.; Zhong, D.F.; Chen, X.Y. Derivatization of $\beta$-dicarbonyl compound with 2,4-dinitrophenylhydrazine to enhance mass spectrometric detection: Application in quantitative analysis of houttuynin in human plasma. J. Mass Spectrom. 2008, 43, 814-824.

19. Yang, N.; Li, Y.J.; Zhou, B.S.; Li, Y.M.; Zhao, W.J.; Liu, J.P.; Li, P.Y. Analysis of chemical compounds of essential oils of Astragalus by SPME-GC-MS. CJTCMP 2018, 33, 3627-3630.

20. Jang, Y.R.; Cho, K.; Kim, S.W.; Altenbach, S.B.; Lim, S.H.; Sim, J.R.; Lee, J.Y. Development of an optimized MALDI-TOF-MS method for high-throughput identification of high-molecular-weight glutenin subunits in wheat. Molecules 2020, 25, $4347-4365$.

21. Zhu, H.L.; Mao, X.B.; Li, L.Y.; Liu, H. Analysis of aristolochie acid a content in asarum volatile oil. J. Southwest China Norm. Univ. Nat. Sci. Ed. 2015, 40, 177-180.

22. Xie, J.; He, J.M. GC-MS The application of gas chromatography-mass spectrometry (GC-MS) in the detection and analysis of volatile oil in plants. J. Shaoguan Univ. Nat. Sci. 2020, 41, 61-65.

23. Xu, R.; Jiang, L.M.; He, J.M.; Liu, Y.L. The condensation mechanism of sodium new houttuyfonate and determination of the chemical structure of condensation products. Acta Pharm. Sin. 2009, 44, 609-614.

24. Xue, Q.S.; Wang, Z.; Yin, R.H. Highly efficient extraction of decanoyl acetaldehyde in Houttuynia cordata Thunb. under steam distillation and its mass spectrometric analysis. J. Anal. Sci. 2012, 28, 28-33.

25. Yi, Y.; Liang, A.H.; Liu, T.; Li, C.Y.; Zhao, Y.; Cao, C.Y. Analysis of causes for adverse reaction of houttuynia injection. China J. Chin. Mater. Med. 2008, 33, 2439-2442.

26. Xue, Q.S.; Yin, H.R. Highly efficient extraction of decanoyl acetaldehyde in Houttuynia cordata Thunb under solvent immersin. J. Anal. Sci. 2013, 29, 386-390.

27. Pang, J.M.; Dong, W.J.; Li, Y.H.; Xia, X.J.; Liu, Z.H.; Hao, H.Z.; Jiang, L.M.; Liu, Y.L. Purification of Houttuynia cordata Thunb. essential oil using macroporous resin followed by microemulsion encapsulation to improve its safety and antiviral activity. Molecules 2017, 22, 293-309.

28. Zwiener, C.; Glauner, T.; Frimmel, F. Method optimization for the determination of carbonyl compounds in disinfected water by DNPH derivatization and LC-ESI-MS-MS. Anal. Bioanal. Chem. 2002, 372, 615-621. [CrossRef]

29. Chi, Y.G.; Feng, Y.L.; Wen, S.; Lv, H.X. Determination of carbonyl compounds in the atmosphere by DNPH derivatization and LC-ESI-MS/MS detection. Talanta 2007, 72, 539-545. [CrossRef]

30. Richardson, S.D.; Caughran, T.V.; Poiger, T.; Guo, Y.B.; Crumley, F. Application of DNPH derivatization with LC/MS to the identification of polar carbonyl disinfection byproducts in drinking water. J. Int. Ozone Assoc. 2000, 22, 653-675. [CrossRef]

31. US Food and Drug Administration. Guidance for Industry: Bioanalytical Method Validation; US Food and Drug Administration: Washington, DC, USA, 2001. 